\title{
Fuzzy sets and real options approaches for innovation-based investment projects effectiveness evaluation
}

\author{
Olga A. Kalchenko ${ }^{1, *}$ \\ ${ }^{1}$ Peter the Great St.Petersburg Polytechnic University, Institute of Industrial Management, Economics \\ and Trade, 195251 Polytechnicheskaya, Saint-Petersburg, Russia
}

\begin{abstract}
The paper focuses on the specifics of innovation-based investment projects effectiveness evaluation (IIPEE). When using traditional approaches, errors occur in evaluation, since innovation-based investment projects are long-term with high uncertainty. Fuzzy sets and real options approaches have long been used as alternative for IIPEE. The novelty of this paper is in the joint use of these two approaches with the consideration of the wide variety of project implementation scenarios. Consequently, it reasonably seems more efficient use of fuzzy sets approach together with Weighted Average Polynomial Option Pricing Model for IIPEE.
\end{abstract}

Key words: fuzzy sets, innovation, investment, effectiveness, evaluation

\section{Introduction}

Innovative changes are essential proactive actions to prevent future negative situations of dynamic environment.

Innovation-based investment projects differ from traditional investment projects by the following features:

- High degree of uncertainty - very often cash flows have irregular nature, i.e. there is no probability distribution of key parameters.

- Problems in strategic effect valuation that can have more qualitative than quantitative character.

- More qualitative than quantitative information on the project, as well as unpredictable risks that have to be taken into consideration.

In case of innovation investment projects, efficient instruments that would allow managing high risks and future uncertainty are urgently needed. Today so far Discounting Cash Flow Method (DCF) with main criterion Net Present Value (NPV) is the most widespread analytical tool for the investments effectiveness evaluation due to calculation simplicity and practical accessibility of the approach.

However, this approach has fundamental inaccuracies concerning IIPEE:

- Ideal market conditions requirements;

\footnotetext{
*Corresponding author: o.kaltchenko@mail.ru
} 
- Investor's flexibility and unpredictable qualitative information flow disregard. DCF method does not take into account any new unexpected market information that arises during the project lifetime. Changes in legislation, sudden competitor's actions, a new technology development, exact experimental results, and others are examples of opportunities and threats that may change investor's behaviour and strategy. Investors can force projects or stop them relying on the new market information [1];

- Inadequate risk calculation. Risk calculation in the denominator by the cumulative discounting rate does not solve the problem of considering high risk in innovation-driven projects. Incorrectness arises by the reason of decreasing value to the present moment not only for cash in-flows, but also for out-flows. This situation is associated with the fact that discounting to the present value gives correct values only if the cash flows sequence is standard [2].

\section{Fuzzy sets approach for IIPEE}

Fuzzy sets approach allows taking into consideration the value of management flexibility, it is proposed to use a slightly modified fuzzy-interval method [3].

The main advantages of fuzzy sets approach are the possibilities:

- to implement multi-criteria evaluation, including both qualitative and quantitative indicators;

- to operate data, the values of which cannot be set explicitly;

- to formalize in a unified form and to use all available heterogeneous information (deterministic, interval, statistical, linguistic), which increases reliability and quality of decisions in selecting innovation projects;

- to take into account the uncertainty of scientific and technical developments;

- to save time on finding exact values of variables and writing equations that describe them (when the decision maker performs the forecast for the initial parameters, it is not required to generate point probabilistic assessment, it is sufficient to specify the calculated range of the predicted parameters);

- to form a sufficiently complete set of possible scenarios of innovation-based investment project development;

- to present the expected development effectiveness not as a single-valued indicator, but as a certain range of values with its expectations distribution, characterized by the membership function of the corresponding fuzzy number. Weighted complete set of expectations allows to get an integrated measure of negative results expectations during the development implementation and to assess the risk level.

According to the fuzzy sets approach [3], the basic parameters of an innovation-based investment project can be evaluated in the form of triangular fuzzy sets (minimum, maximum, and most expected values) and, for each level of confidence (from 0 to 1), construct interval evaluation of net present value (NPV):

$\left[N P V_{1}, N P V_{2}\right]=\left[-l_{2}+\sum_{i=l}^{N} \frac{\Delta V_{i l}}{\left(1+r_{i 2}\right)^{i}}+\frac{c_{l}}{\left(1+r_{N+1,2}\right)^{N+1}}-l_{l}+\sum_{i=l}^{N} \frac{\Delta V_{i 2}}{\left(1+r_{i l}\right)^{i}}+\frac{c_{2}}{\left(1+r_{N+1,1}\right)^{N+1}}\right]$

where $\left[\mathrm{I}_{1}, \mathrm{I}_{2}\right]$ - the range of possible values of the initial investment volume, $\mathrm{N}$ - the number of planned intervals (periods, stages) of the investment process, $\left[\Delta \mathrm{V}_{\mathrm{i} 1}, \Delta \mathrm{V}_{\mathrm{i} 2}\right]-$ interval working balance of receipts and payments in the i-th period, $\left[\mathrm{r}_{\mathrm{i} 1}, \mathrm{ri}_{2}\right]$ - interval discount rate, $\left[\mathrm{C}_{1}, \mathrm{C}_{2}\right]$ - liquidation value of the net assets, established during the investment process, $(\mathrm{N}+1)$ - interval, not related to the life of the project, but the time of completion of the settlement of all innovation project participants [4]. 


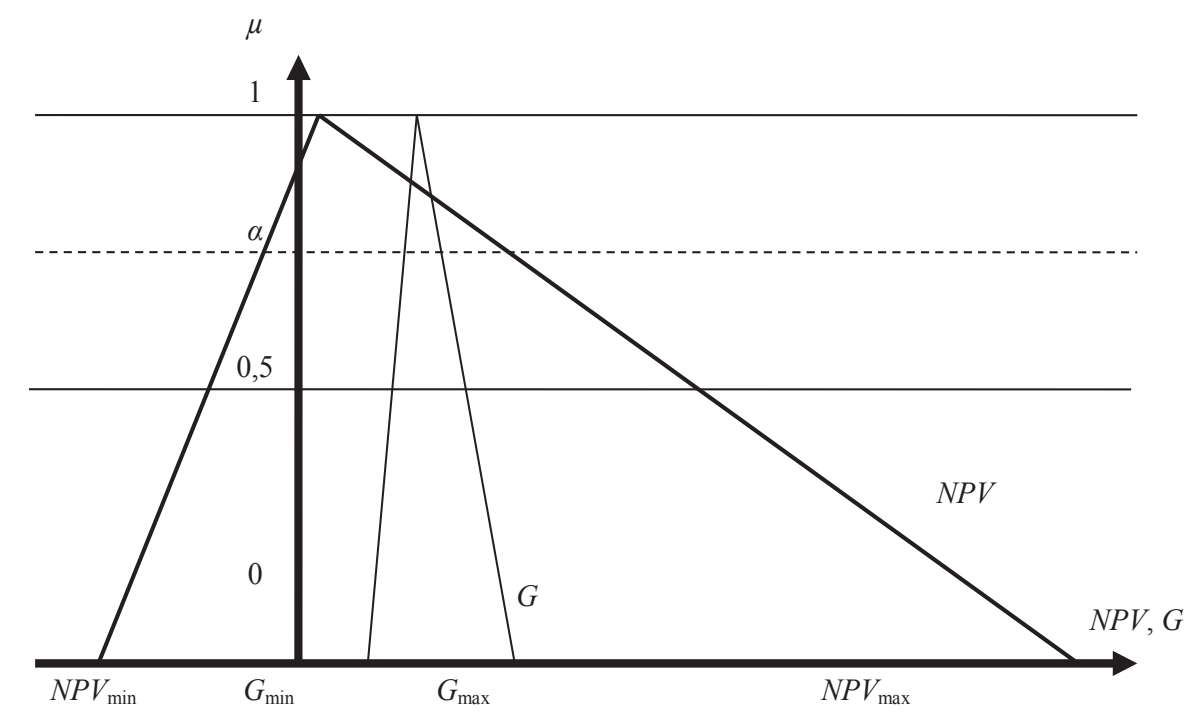

Fig. 1. NPV fuzzy interval evaluation [4]

The project is considered effective when the NPV is more than a certain G level (in the common case $\mathrm{G}=0$ ). This parameter is also a subject of fuzzy sets evaluation. $\mathrm{G}$ indicator is a return requirement for investments.

Given an acceptable level of sampling at the interval $\alpha[0,1]$, it is possible to reconstruct the resulting fuzzy number NPV by approximating its membership function $\mu$ broken curve on interval points. Often, it is possible to bring the NPV to the triangularterm, being limited by calculations of the main points of the outcome data fuzzy numbers. As the performance phases of the project are realized, further information results, allowing refinement and better adjustment of the NPV fuzzy evaluation.

After each stage, as additional information becomes available for the analysis of the innovation project and for possible uncertainty reduction, the fuzzy profile of the project is rebuilt and the risk is recalculated.

\section{Real options approach for IIPEE}

Basic real options - those that have the greatest impact on the implementation of the project, which received the most important decisions to minimize losses and give companies management important information.

In our earlier studies [4], we wrote about the possibility of applying real options approach for IIPEE:

- Practical implementation of classical probabilistic analysis (multi-scenario "decision tree") to the investment evaluation using real options approach with a large number of control points is quite problematic, both due to the complexity of probabilities estimation, and due to the lack of a clear methodology for NPV changes indicators measurement;

- Application of Cox-Ross-Rubinstein Binomial Option Pricing Model (BOPM) [5]. BOPM is based strictly on the binomial changes assumption. For innovation and investment 
projects evaluation is limited, because the future uncertainty is reduced only to two states: positive and negative. However, researchers still have to accept it.

- Scope of Black-Scholes Option Pricing Model (BSOPM) [6] for real investments evaluation is possible when the project success is strongly related to the price of an actively traded liquid asset. For example, the price of oil or other popular resource, when the volatility statistics can meet the requirements. BSOPM is based on the continuous time assumption. This implies a possibility to sell or buy a share at any moment like at the Stock Exchange. BSOPM is based on the replicated portfolio assumption. Innovation market is developing, and there are no statistics on it. An expert evaluation of volatility, taking into account external and internal company risks, is required.

The following conclusions were drawn: these approaches are based either on subjective probabilities or tied to market indicators, therefore, the possibilities of their application to IIPEE are limited. Innovation-based investment projects are characterized by significant uncertainty, large number of decision points and weak predictability of environmental parameters, therefore none of these methods of applying the real options approach can be used to evaluate effectiveness. However, the real options approach provides an opportunity to develop an algorithm for decision-making in projects implementation.

We proposed to apply real options approach for IIPEE in two ways:

1) quantitative interpretation of an option and determination of its present value, either on the basis of outcomes probabilities estimation, or as a link to some liquid replicable underlying asset;

2) option is a mechanism for constructing decision algorithms, a qualitative understanding of options as a resource of flexibility.

Innovation-based investment projects can be considered real options, because, in the initial stages the shortage and unreliability of financial information occurs. Such projects can be stopped with a much smaller financial loss. The consideration is aimed at identifying their additional features and capabilities by:

- varying project parameters during implementation;

- quantitative assessment of the available options and their inclusion in the project cost.

As previously mentioned, real options approach allows overcoming the mentioned problems:

1. investors' flexibility valuation;

2. risk calculation in the numerator by the scenarios (or decision) tree.

The idea of Real Option Valuation (ROV) was taken from the stock market where investor's opportunity, but not the obligation to sell or to buy an asset, is valued.

S. Myers was the first, who implemented option finance technique to the real investment [7]. Nowadays the leading specialists in the real option valuation significantly contributed to this area [1, 8-10].

E. Rogova and A. Yarygin suppose that the plenty of researches devoted to the ROV method may be divided into two parts [2]:

1. strong mathematic papers that sometimes do not give the clear way of using results in practice $[11,12]$;

2. papers devoted to method popularization [13].

On the basis of these models pitfalls, E. Rogova and A. Yarygin developed Weighted Average Polynomial Option Pricing Model (WAPOPM). Since BOPM does not need to estimate volatility parameter $(\sigma)$ and is based on the discrete time assumption, it is considered as a basic method in their research. The aim of their research was to construct a decision tree with any possible complex structure (any time-intervals between the project's stages, and any amount of the scenarios at each stage) [2].

Each specific innovation-based investment project leads to corresponding decision tree. 
Instead of searching the formula for real option value at the initial moment of time in all possible cases, they propose a unique analytical algorithm of calculation in any subtree (part of the tree constructed from parent node and its children nodes).

After real option value calculation at the leafs, ROV at the parent node of these leafs is calculated. The goal is the ROV evaluation at the root.

An innovation-based investment project is considered to have three possible scenarios: successful; non-profitable and breakeven; detrimental.

In case of two possible ways, BOPM algorithm is used, which is based on the equal portfolio value assumption regardless of the way (basic asset price change). The portfolio consists of basic asset, risk-free obligations, and an option on them. Cox-Ross-Rubinstein's remark is [5]: "from either the hedging or complete markets approaches, it should be clear that three-state or trinomial stock price movement will not lead to an option pricing formula based solely on arbitrage considerations."

In the work of E. Rogova and A. Yarygin valuation for call-option and for put-option is proposed.

Strategic NPV of innovation-based investment project equals to:

Expanded (strategic) $N P V=$ direct (passive $N P V)+$ Real Options Value (flexibility value)

Their unique analytical calculations algorithm in any subtree includes:

1. Technological input data identification (a decision tree which reflects particularities of the innovation-based investment project, time-intervals between the project's stages).

2. Financial input data identification (risk-free rate, basic asset price at the initial moment of time, option exercise price).

3. Parameters that reflect change in basic asset price identification (fuzzy sets approach that was proposed earlier in our research is applicable, as innovation-based investment project are often characterized by poor statistic data).

4. Leafs ROV calculation.

5. Root ROV evaluation by iteratively using WAPOPM in all subtrees.

The valuation of the real options in innovation-based investment projects with extended uncertainty is an important practical problem.

WAPOPM:

- takes into account investors' flexibility and it calculates investment risk by the scenarios tree (decision tree);

- does not need an estimation of the volatility parameter and it is based upon the discrete time assumption;

- is based on the polynomial changes;

- can be easily used in practice together with fuzzy sets approach.

\section{Conclusions}

In our previous research we proposed and approved the use of fuzzy sets approach together with real options as quantitative assessment and project parameters variation for IIPEE [4].

Presently, according to recent development [2], it reasonably seems more efficient use of fuzzy sets approach together with WAPOPM for IIPEE.

Fuzzy sets and real options approaches for IIPEE allow constant critical analysis and the consideration of alternative parameters in the internal and external environment. It becomes possible not to evaluate the entire project at once, but divide it into several stages as investor opportunities are acquired. Fuzzy sets and real options approaches allow evaluating the efficiency and choosing between alternatives without using other methods, by considering the relationship between risk and opportunities that companies face. 
It becomes possible to correct innovation-based investment projects during their realization. The ability to manage innovation-based investment projects is achieved by the joint use of fuzzy sets and real options approaches.

Fuzzy sets and real options approaches for IIPEE were used and approved at three different sized industrial companies, situated in one of the most innovative region of Russia - Saint-Petersburg and Leningrad region. The first is a medium-sized company, JSC "Ecoenergetika," which is the developer and owner of advanced Russian technology in the field of energy saving. The second is a medium-sized agro-industrial company of Leningrad region. The third is a large-sized organization, JSC "NPP" Radar MMS," which is a world leader in the field of electronics, special and civil systems, precise instruments, and special software.

It is very important and interesting to scrutinize in further calculations improvement on the basis of a software product, for example Matlab Fuzzy Logic Toolbox.

Author is thankful to Mr. Prof. Dr. Vasiliy R. Okorokov for his guidance and valuable comments. All errors are the sole responsibility of the author.

\section{References}

1. V. Antikarov, T. Copeland, Real options: a practitioner's guide, Texere (2001)

2. E. Rogova, A. Yarygin, Valuing innovative 4G (LTE) technology with real options approach. The XXIV ISPIM Conference Proceedings (2013)

3. A. O. Nedosekin, The applications of fuzzy sets theory to problems of finance control. Audit and financial analysis, 2, 15-18 (2000)

4. V. R. Okorokov, O. A. Kalchenko, An innovative project evaluation technique under conditions of information uncertainty. International Journal of Business and Information, 10, 180-197 (2015)

5. J. Cox, S. Ross, M. Rubinstein, Option pricing: A simplified approach. Journal of Financial Economics, 7(3), 229-263 (1979)

6. F. Black, M. Scholes, The pricing of options and corporate liabilities. The Journal of Political Economy, 81(3), 637-654 (1973)

7. S. Myers, Determinants of corporate borrowing. Journal of Financial Economics, 5(2), 147-175 (1977)

8. L. Trigeorgis, Strategic Investment, Princeton University Press (2004)

9. A. Damodaran, The Promise and Peril of Real Options. Working paper, Stern School of business (1999)

10. J. C. Hull, Options, Futures and Other derivatives, Prentice Hall (2002)

11. S. M. Turnbull, Option Valuation, Dryden Press (1987)

12. P. Wilmott, S. Howison, J. Dewynne, The Mathematics of Financial Derivatives, Cambridge Univ. Press (1995)

13. K. J. Leslie, M. P. Michaels, The real power of real options. The McKinsey Quarterly, (3), 5-22 (1997)

14. H. Smit, L. Trigeorgis, Real Options: Examples and principles of valuation and strategy, Oxford University Press (2003) 